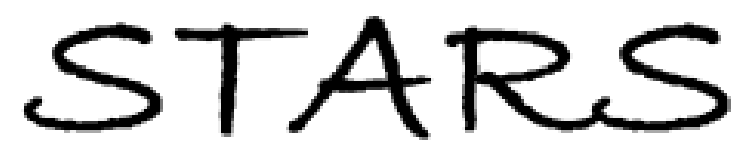

University of Central Florida

STARS

Faculty Bibliography 1990s

Faculty Bibliography

$1-1-1999$

\title{
Broad band p-Ge optical amplifier of terahertz radiation
}

\author{
A. V. Muravjov \\ University of Central Florida \\ S. H. Withers \\ University of Central Florida \\ S. G. Pavlov \\ V. N. Shastin \\ R. E. Peale \\ University of Central Florida
}

Find similar works at: https://stars.library.ucf.edu/facultybib1990

University of Central Florida Libraries http://library.ucf.edu

This Article is brought to you for free and open access by the Faculty Bibliography at STARS. It has been accepted for inclusion in Faculty Bibliography 1990s by an authorized administrator of STARS. For more information, please contact STARS@ucf.edu.

\section{Recommended Citation}

Muravjov, A. V.; Withers, S. H.; Pavlov, S. G.; Shastin, V. N.; and Peale, R. E., "Broad band p-Ge optical amplifier of terahertz radiation" (1999). Faculty Bibliography 1990s. 2757.

https://stars.library.ucf.edu/facultybib1990/2757

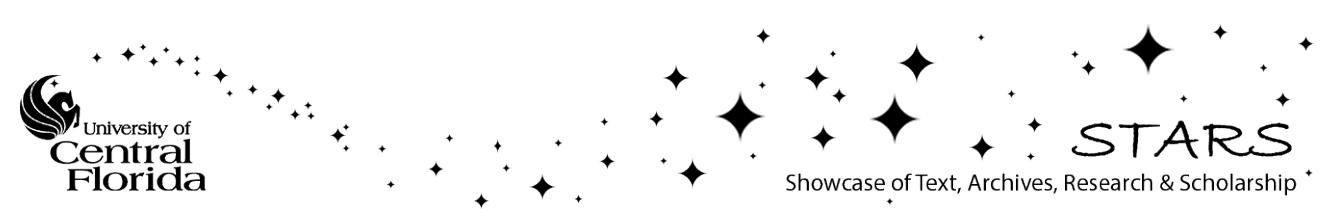




\section{Broad band $p$-Ge optical amplifier of terahertz radiation}

Cite as: Journal of Applied Physics 86, 3512 (1999); https://doi.org/10.1063/1.371250

Submitted: 10 May 1999 . Accepted: 10 June 1999 . Published Online: 20 September 1999

A. V. Muravjov, S. H. Withers, S. G. Pavlov, V. N. Shastin, and R. E. Peale

Lock-in Amplifiers ... and more, from DC to $600 \mathrm{MHz}$

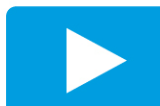

Watch 


\title{
Broad band $p$-Ge optical amplifier of terahertz radiation
}

\author{
A. V. Muravjov and S. H. Withers \\ Department of Physics, University of Central Florida, Orlando, Florida 32816
}

S. G. Pavlov and V. N. Shastin

Institute for Physics of Microstructures, Russian Academy of Sciences, GSP-105, Nizhny Novgorod 603600, Russia

R. E. Peale ${ }^{\mathrm{a})}$

Department of Physics, University of Central Florida, Orlando, Florida 32816

(Received 10 May 1999; accepted for publication 10 June 1999)

\begin{abstract}
A solid state broad band amplifier of terahertz radiation $(1.5-4 \mathrm{THz})$, based on intersubband transitions of hot holes in $p$-Ge is demonstrated. The gain is investigated as a function of applied magnetic and electric fields by transmission measurements using a laser system with two $p$-Ge active crystals, when one operates as an oscillator and one as an amplifier. A peak gain higher than usually reported for $p$-Ge lasers has been achieved using time separated excitation of the oscillator and amplifier. Distinct differences in gain dependence on applied fields are noted between low- and high-frequency modes of $p$-Ge laser operation. (C) 1999 American Institute of Physics.
\end{abstract}

[S0021-8979(99)04718-0]

\section{INTRODUCTION}

The mechanism of amplification of terahertz emission in bulk $p$-Ge is based on direct optical transitions between light and heavy hole valence subbands (Fig. 1) in strong crossed electric and magnetic fields, when the crystal is cooled to liquid helium temperatures. ${ }^{1}$ The population inversion builds up for certain ratios of electric and magnetic fields, when light holes are accumulated on closed trajectories below the optical phonon energy, while heavy holes are strongly scattered by optical phonons. Due to the wide frequency range of light to heavy hole transitions, the amplification has a broad spectrum with $\delta \omega / \omega \sim 1$, and therefore, this active medium is appropriate for propagation and amplification of picosecond pulses of terahertz radiation.

Traditional $p$-Ge lasers cover the frequency range 1.5$4.2 \mathrm{THz}$, have a $1-10 \mathrm{~W}$ peak output power for $1 \mathrm{~cm}^{3}$ typical active volume, and have a 1-5 $\mu$ s laser pulse duration. The saturation intensity of far-infrared radiation inside the active $p$-Ge crystal can reach $\mathrm{kW} / \mathrm{cm}^{2}$, but this intensity is unachievable at the laser output because of the typical gain value of only about $10^{-2} \mathrm{~cm}^{-1}$, which requires small outcoupling losses for the development of stimulated emission.

A possible method of increasing the useable $p$-Ge laser power is a combined oscillator-amplifier $p$-Ge laser system. Since a single-pass amplifier does not require feedback from an out-coupling mirror, the physical limit for available output intensity increases to the full $\mathrm{kW} / \mathrm{cm}^{2}$ saturation intensity achievable within the active amplifier crystal. In this work, the use of an active $p$-Ge crystal as an amplifier for farinfrared radiation is demonstrated. The gain in the amplifier crystal was studied as a function of applied fields. The peak gain in the amplifier was found to be higher than the small signal gain usually reported for $p$-Ge lasers.

\footnotetext{
${ }^{a)}$ Electronic mail: rep@physics.ucf.edu
}

\section{EXPERIMENT}

The oscillator-amplifier scheme is shown in Fig. 2. Rectangular rods were cut from single crystal Ge, doped by Ga with a concentration $N_{A}=7 \times 10^{13} \mathrm{~cm}^{-3}$. The ends of the crystals were polished and made parallel to each other with 30 arc sec accuracy. The laser crystal is $28 \mathrm{~mm}$ long and the amplifier crystal is $67 \mathrm{~mm}$ long. Si spacers between the crystals prevented electrical breakdown. The out-coupling mirror for the laser was made by an evaporated $\mathrm{Al}$ film on one of the Si spacers with a $1.5 \mathrm{~mm}$ output hole in the center. The back copper mirror was attached to the back end of the laser crystal via $20 \mu \mathrm{m}$ teflon film. Electric field pulses $E_{1}$ and $E_{2}$ were applied to the laser and amplifier crystals from separate pulsers via ohmic $\mathrm{Al}$ contacts evaporated on the lateral sides of the crystals. The entire system was inserted in a superconducting solenoid, so that the applied magnetic field $B$ was the same for the oscillator and amplifier crystals, and cooled by liquid helium. The radiation was detected by a whiskercontacted Schottky diode outside the cryostat or with a cooled Ge:Ga photoconductor inside the cryostat.

\section{RESULTS}

The dependence of the gain on the applied electric field in the "low" and "high" frequency regions of $p$-Ge amplification were studied separately. To clarify the distinction between these two regimes, we present laser emission spectra. Figure 3a shows a typical spectrum ${ }^{1,2}$ of $p$-Ge laser emission in the high frequency band for a magnetic field value of $0.7 \mathrm{~T}$ and an electric field of $1 \mathrm{kV} / \mathrm{cm}$. Here the laser generates a broad multimode spectrum in the range $70-100 \mathrm{~cm}^{-1}$. When $E$ and $B$ are increased, this band shifts to higher frequency up to the upper border of $140 \mathrm{~cm}^{-1}$. 


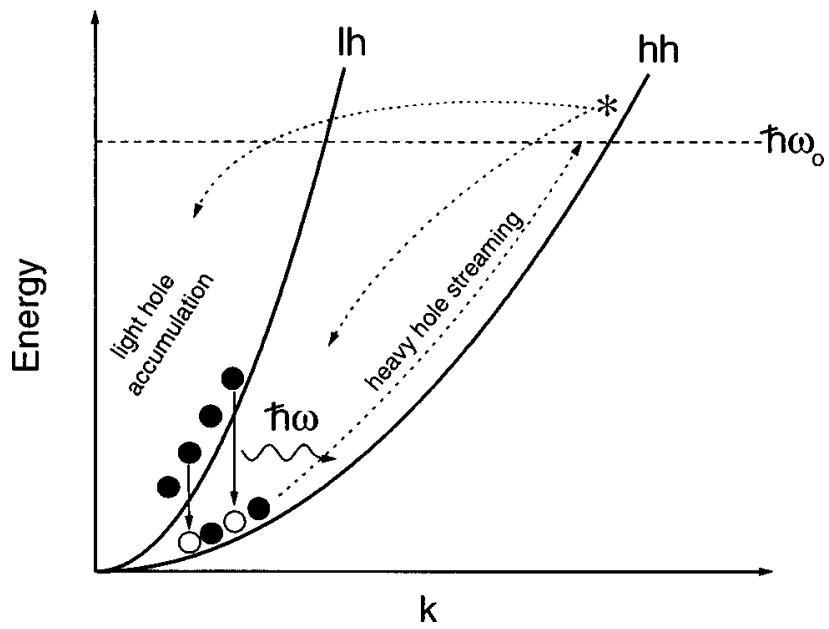

FIG. 1. Mechanism of terahertz amplification by intersubband transitions. Solid parabolas represent the light- (lh) and heavy-hole (hh) bands. The dotted lines represent heavy holes accelerated beyond the optical phonon energy $\left(\hbar \omega_{o}\right)$, then scattered back to the heavy- or light-hole band. Accumulation of hot light holes is indicated together with downward transitions and terahertz photon emission.

Figure 3(b) shows a spectrum in the low frequency region for fields of $E=670 \mathrm{~V} / \mathrm{cm}$ and $B=0.42 \mathrm{~T}$. Figure 3(b) is Fourier spectrometer data collected by the Event-locked method $^{3}$ at a resolution of $0.04 \mathrm{~cm}^{-1}$. This spectral region of $p$-Ge laser emission is always characterized by sharp lines that are associated with transitions between acceptor bound states. $^{2}$ These lines move not more than $0.5 \mathrm{~cm}^{-1}$ with changes in fields. Although their relative intensities may change, the line at $54.5 \mathrm{~cm}^{-1}$, which nearly coincides with the Ga acceptor absorption " $G$ ', line, usually dominates. ${ }^{2}$ The inset in Fig. 3, shows that longitudinal mode structure is resolved for this $28 \mathrm{~mm}$ long laser crystal, which was used in these experiments as the laser oscillator (see Fig. 2).

The transmission $T$ of oscillator radiation by the amplifier measured in the "low frequency" operation range ${ }^{1}$ of the $p$-Ge laser at field values $B=0.47 \mathrm{~T}$ and $E_{1}=700 \mathrm{~V} / \mathrm{cm}$ is presented in Fig. 4 as a function of electric field $E_{2}$ applied

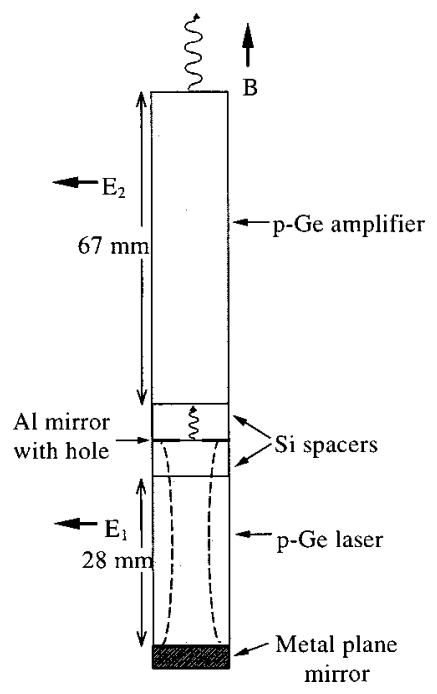

FIG. 2. Oscillator-amplifier $p$-Ge laser construction.

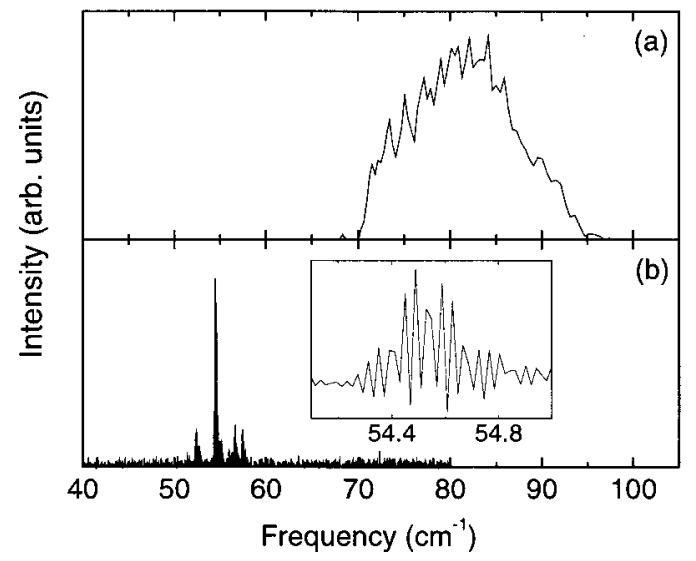

FIG. 3. High (a) and low (b) frequency regions of $p$-Ge laser emission. The applied fields are (a) $0.7 \mathrm{~T}$ and $1 \mathrm{kV} / \mathrm{cm}$, and (b) $0.42 \mathrm{~T}$ and $670 \mathrm{~V} / \mathrm{cm}$. The inset shows resolved longitudinal mode structure within the $54.5 \mathrm{~cm}^{-1}$ impurity-related $G$ line. The length of the active $p$-Ge crystal was $28 \mathrm{~mm}$.

to the amplifier crystal. The crystal is almost transparent when $E_{2}=0$. When an electric field $E_{2}$ is applied, the tranmission drops to zero. Further increase of $E_{2}$ causes $T$ to increase again and reach a maximum at around $600 \mathrm{~V} / \mathrm{cm}$. Note that a $20 \%$ shot-to-shot intensity instability, characteristic of $p$-Ge laser operation in the low frequency regime and indicated in Fig. 4 by the error bar, prevents absolute determination of the transmission from these measurements. Nevertheless, the evidence that gain in the amplifier crystal overcomes at least most of the losses is clear.

Figure 5 presents the very different behavior of the amplifier when the laser operates in the high frequency regime. The laser oscillator is excited by a field $E_{1}=800 \mathrm{~V} / \mathrm{cm}$, and both crystals are in a magnetic field $B=0.69 \mathrm{~T}$. For low excitation of the amplifier $\left(E_{2}<20-50 \mathrm{~V} / \mathrm{cm}\right)$, there is no transmission of oscillator radiation, but for higher fields, transmission appears. For electric field values $E_{2}$ in the range $800-1200 \mathrm{~V} / \mathrm{cm}$ the amplifier enters the active zone of amplification on 1-h transitions due to light-hole accumulation.

To define the amplifier zero-gain level $(\mathrm{T}=1)$ in Fig. 5, the threshold electric fields of self-excitation were independently determined for the amplifier crystal with external mirrors applied to its ends (i.e., when it starts to lase). Because

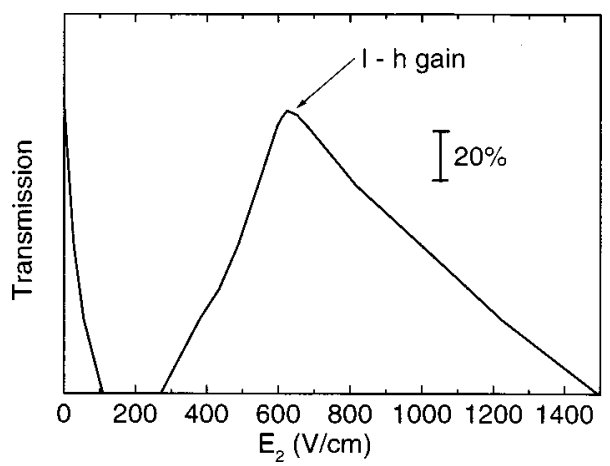

FIG. 4. Transmission of low-frequency-domain $p$-Ge laser radiation by the $p$-Ge amplifier crystal vs excitation field $E_{2}$. The spectral range of the emission is $51-58 \mathrm{~cm}^{-1} . E_{1}=700 \mathrm{~V} / \mathrm{cm}$ and $B=0.47 \mathrm{~T}$. 


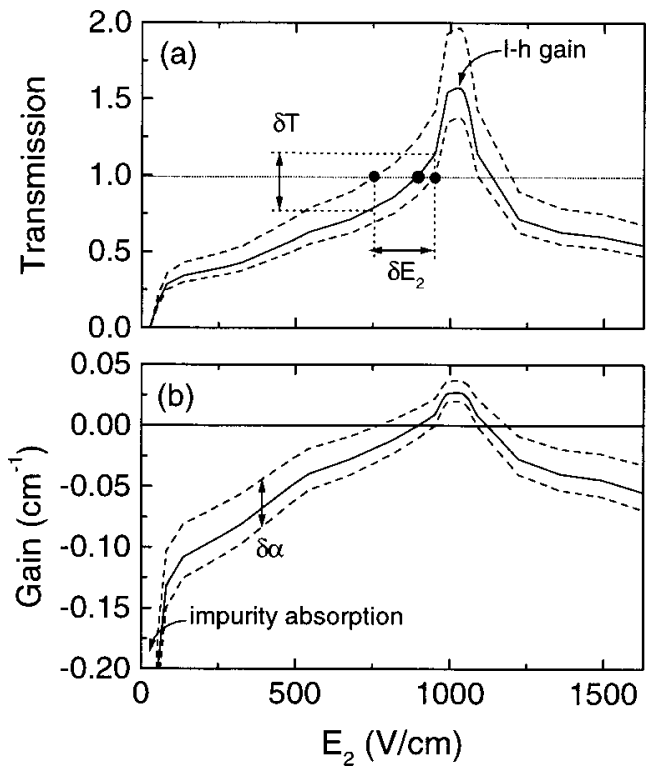

FIG. 5. (a) Transmission of high-frequency-domain $p$-Ge laser radiation by the $p$-Ge amplifier vs excitation field $E_{2}$. The dotted horizontal line indicates $100 \%$ tranmission. (b) Gain in the amplifier crystal determined from transmission data. The horizontal solid line indicates zero net gain. In both figures, the bounding dashed data curves indicate the range of uncertainty. The spectral range of the emission is $80-100 \mathrm{~cm}^{-1} . E_{1}=800 \mathrm{~V} / \mathrm{cm}$ and $B$ $=0.69 \mathrm{~T}$.

the threshold might depend on the mode structure, maximum and minimum estimated threshold levels for that sample, giving the range $\delta E_{2}$, are marked on Fig. 5. These levels define the range of values for transmission $\delta T$ (dashed curves) and for gain $\delta \alpha$.

Accounting for multiple reflections inside the amplifier crystal (considering $100 \%$ reflection from the back $\mathrm{Al}$ mirror and $R=(n-1)^{2} /(n+1)^{2}=0.35$ from the output end, $n_{\mathrm{Ge}}$ $=3.925)$, the absolute value of the gain is found from the expression $T=g(1-R) /\left(1-R g^{2}\right)$, where $g=\exp (\alpha L)$ and $L$ is length of the crystal. The gain $\alpha$ obtained from the peak in Fig. 3(a) has the value $0.028(+/-0.008) \mathrm{cm}^{-1}$. Note that the measurement of the absolute gain from the rise time of the stimulated emission pulse when external mirrors are applied to the crystal gives the lower value $0.010-0.015 \mathrm{~cm}^{-1}$.

The enhanced amplifier gain has been reached by delaying $E_{2}$ with respect to the $E_{1}$ pulse, as shown in Fig. 6. In this way, the amplifier is switched on when the oscillator emission has already reached its maximum intensity. The laser emission shown in Fig. 6 is detected with a Ge:Ga photoconductor inside the cryostat in close proximity to the cavity construction, so that the detector can measure oscillator emission that has not been transmitted by the amplifier due to rays that leak out of the laser cavity and are scattered inside the cryostat. The 400 ns delay in Fig. 6 between the onset of oscillator excitation $E_{1}$ and the oscillator emission pulse (solid curve) is typical for the build-up time of $p$-Ge lasers. If the amplifier is not excited, the solid curve shows the usual decay caused by crystal heating. On the other hand, if the amplifier receives an excitation pulse $E_{2}$ when the oscillator signal is already present, the signal output from the

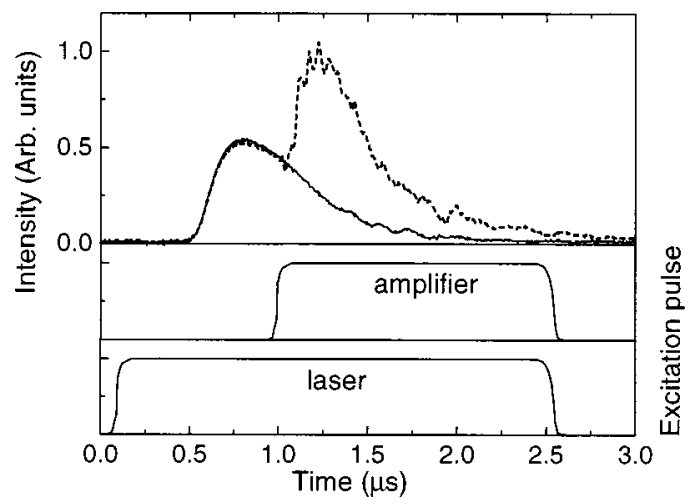

FIG. 6. Timing diagram of oscillator excitation pulse $E_{1}$, amplifier excitation pulse $E_{2}$, oscillator output pulse (solid data) and amplifier output pulse (dashed data). The radiation is measured within the cryostat by the Ge:Ga detector, which is also sensitive to scattered oscillator radiation even when the amplifier crystal is opaque.

combined system rises immediately, with essentially no delay from the onset of $E_{2}$ (dashed curve).

To measure the radiation outside the cryostat, we used a room temperature fast whisker-contacted Schottky diode, which detects only the second peak of the dashed curve in Fig. 6. This second peak corresponds to a highly collimated beam passed through the amplifier crystal only while the amplifier is active. When $E_{2}=0$, the amplifier crystal is not transparent, and the scattered signal from the oscillator is strongly attenuated by reflections within the light pipe.

A fragment of the signal detected by the Schottky diode (Fig. 7) shows fast periodic structure with 800 ps period, which corresponds to round trip oscillations of the oscillator laser cavity (28 mm active Ge plus $4 \mathrm{~mm} \mathrm{Si} \mathrm{spacer).} \mathrm{This}$ periodic pattern is undistorted by dispersion or multiple reflections inside the amplifier, which demonstrates that the second active crystal works similarly to a single pass amplifier and that this amplifier reproduces the subnanosecond dynamics of the laser intensity. This observation, and the known broad gain spectrum, suggests that the $p$-Ge amplifier is suitable for propagation and amplification of pulses of terahertz radiation even as short as 1 ps duration.

\section{DISCUSSION AND CONCLUSION}

The difference between low-field and high-field transmission data revealed in Figs. 4 and 5 is explained as follows. When $E_{2}=0, p$-Ge laser radiation in the high frequency region has sufficiently high photon energy to ionize Ga impurities within the amplifier crystal, but low frequency radiation $\left(51-58 \mathrm{~cm}^{-1}\right)$ cannot. We assume that $G$-line absorption is free from Stark broadening $\left(E_{2}=0\right)$ and is much narrower than the $G$-related emission band of the $p$-Ge laser shown in Fig. 3. Hence, the amplifier crystal is lossy for the high-frequency $p$-Ge laser radiation but almost transparent for low-frequency radiation. The decrease of $T$ for lowfrequency radiation observed in Fig. 4 at small $E_{2}$ values is likely due to Stark broadening of the $G$-line into the lowfrequency laser spectral range and the appearance of free carrier absorption. In Fig. 5, the transmission appears at relatively small values of $E_{2}$ when this field is sufficient to 
nearly empty all impurity states by impact ionization and the high frequency oscillator radiation can bleach any residual impurity absorption. Free carrier absorption, which is proportional to $\omega^{-2}$, should be less of a contribution in the high frequency region. In both Figs. 4 and 5, the transmission rises at sufficiently high $E_{2}$ due to gain on direct optical light-to-heavy hole transitions, in correspondence to the optimal ratio $E_{2} / B \sim 1.4 \mathrm{kV} / \mathrm{T} \mathrm{cm}{ }^{1}$

The amplifier-transmission results presented here are in good agreement with the experiments by Keilmann and Zuckermann, ${ }^{4}$ where the transmission through an active $p$-Ge crystal of far-infrared gas-laser pulses was studied. Keilmann and Zuckermann similarly reported that the small signal gain determined from the amplifier transmission is higher than the value obtained from the risetime of $p$-Ge lasing. The reason is that the typical build-up time of the radiation intensity from the spontaneous level to a measurable level is several hundred nanoseconds. Meanwhile, the sample undergoes Joule heating from 4 to about $10 \mathrm{~K}$, where the absolute gain is reduced by the decreased light-hole lifetime and increased multiphonon lattice absorption. (Lasing typically shuts down completely at about $20 \mathrm{~K}) .^{5}$ The combined oscillator/ amplifier $p$-Ge laser reported here avoids this problem by delaying the amplifier excitation until the oscillator has reached its maximum output. In this way, the amplifier crystal is still cold when the injected radiation reaches its peak intensity, allowing this radiation to be amplified with higher gain than in usual $p$-Ge lasers. This method will in principle allow higher output intensities for the same excited volume of $p$-Ge crystal than usually found.

A conclusion from this work is the potential for application of $p$-Ge laser active crystals as solid state amplifiers of

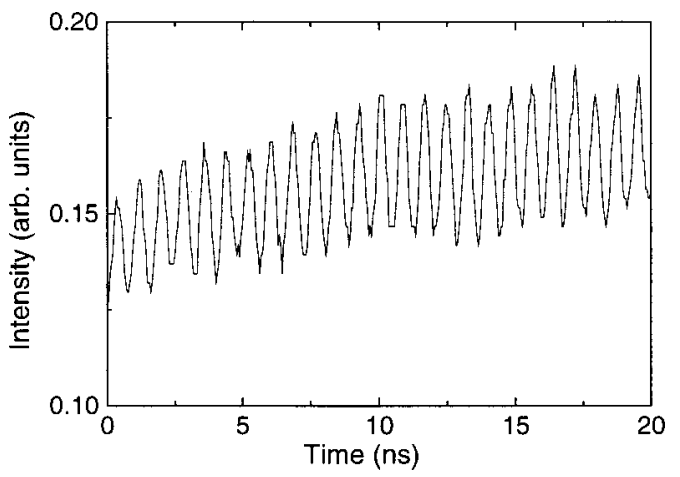

FIG. 7. Fast dynamics of the $p$-Ge amplifier output. Subnanosecond round trip intensity oscillations of the $p$-Ge laser oscillator are preserved by passage through the amplifier.

far-infrared radiation in the 1.5-4 $\mathrm{THz}$ spectral range. The broad-band nature of the $p$-Ge amplification may hold promise for amplification of femtosecond terahertz pulses created using ultrafast lasers and photoconductive antennas.

\section{ACKNOWLEDGMENTS}

This work was supported by NSF and BMDO. The coauthors from IPM thank the Russian Foundation for Basic Research.

${ }^{1}$ E. Gornik and A. A. Andronov (eds.), Opt. Quantum Electron. 23, S111 (1991)

${ }^{2}$ S. V. Demichovsky, A. V. Muravjov, S. G. Pavlov, and V. N. Shastin, Semicond. Sci. Technol. 7, B622 (1992).

${ }^{3}$ H. Weidner and R. E. Peale, Appl. Spectrosc. 51, 1106 (1997).

${ }^{4}$ F. Keilmann and H. Zuckermann, Opt. Commun. 109, 296 (1994).

${ }^{5}$ K. Park, R. E. Peale, H. Weidner, and J. J. Kim, IEEE J. Quantum Electron. 32, 1203 (1996). 